\title{
1-Periodic Nanostructures
}

\author{
Mircea V. Diudea, ${ }^{\text {a* }}$ Virginia R. Bucila, ${ }^{a}$ Davide M. Proserpio ${ }^{b}$ \\ ${ }^{a}$ Faculty of Chemistry and Chemical Engineering, \\ “Babes-Bolyai" University, Arany Janos Str. 11, 400028, Cluj, Romania \\ diudea@gmail.com \\ ${ }^{b}$ Università degli Studi di Milano, Dipartimento di Chimica, Via Golgi 19, 20133 Milano
}

(Received April 1, 2013)

\begin{abstract}
Triply periodic structures are the usual subjects of crystallographic studies while the objects of these are the crystals or reticulations. There are amorphous materials with no ordered atomic arrays and some ordered structures with no translational periodicity, eventually called quasicrystals. This study presents a variety of five-fold symmetry molecular networks with 1-periodicity. The construction and topology (the genus calculation included) of these structures is described in terms of the net parameters, in a crystallographic manner.
\end{abstract}

\section{Introduction}

Polymorphism is maybe the most general property of the matter, in its appearance, while its causes are multiple, with the geometry and involved symmetry playing an essential role. The long-range order in crystals is described by the translational symmetry, considering the crystal (or better, the corresponding network) is infinite. However, a crystal is finite and often imperfections/defects appear, so that the border between crystal and amorphous is rather diffuse.

Short-range order of matter was described to appear in quasicrystals, associated with rotational symmetry, not allowed in classical (translational) crystallography. High quality stable quasicrystals, with typical features of crystalline phases, have been isolated and their structure established. The Nobel prize for quasicrystals in 2011 came to recognize the importance of this kind of ordered matter; it can be either a dense or a spongy one. 
In respect of dense structures, we have to address to the well-known problems of space filling by polyhedral cells [1-5] and sphere-packing [6-12]. According to Ulam conjecture [13], all hard convex shapes pack more densely than the spheres, which have a maximum packing fraction of $\varphi=\pi / \sqrt{ } 18 \approx 0.7405$. This happens in the face-centered cubic $f c c$ array and was conjectured by Kepler but only recently demonstrated by Hales [14].

Spongy structures, like zeolites and the synthesized spongy carbon, represent atomic arrangements showing large hollows and/or channels or tubes. Substructures of such materials can be viewed as units of negative curvature, eventually belonging to the well-defined triply periodic minimal surfaces: P, D and G [15]. The graphs associated to these surfaces are called labyrinth graphs [16]. Since a hollow can be viewed as a simple torus (i.e. a hyper-ring, of which edges are tubes), Diudea [17] termed such spongy structures as multi-tori.

Intersection-free triply periodic minimal surfaces are of unbounded genus but can be characterized by calculating the genus per unit cell " $u c$ " [15], defined in terms of the integral Gaussian curvature according to the Gauss-Bonnet [18]

$$
\chi_{u c}=(1 / 2 \pi) \iint_{u c} k \cdot d a
$$

with $\chi_{u c}$ being the Euler-Poincaré characteristic per unit cell [19]. From this, the surface genus [20] is calculated by:

$$
g=1-\chi_{u c} / n
$$

where $\mathrm{n}=1$ for non-orientable (Moebius) surfaces and $\mathrm{n}=2$ for orientable surfaces.

Multi-tori MT are structures of high genera (see below) and are supposed to result by self-assembly of some repeating units (i.e., monomers) which can be designed by opening of cages/fullerenes or by applying appropriate map/net operations on small polyhedral objects, usually the Platonic solids. They can be encountered in zeolites [21], natural or synthetic aluminosilicates with an open/spongy three-dimensional crystal structure.

The article is structured as follows. After the introductory part, some basic map operations used in the building of repeating units of periodic structures are presented. In the third part the new 1-periodic networks are discussed. The genus calculation in such quasicrystals is given in the forth's section. Conclusions and references will close the paper. 


\section{Operations on Maps}

Design of the structures herein discussed is based on "operations on maps", merely applied on the Platonic solids: tetrahedron (T), cube (C), octahedron (Oct), dodecahedron (Do) and icosahedron (Ico). A map M is a discretized surface domain while the operations on maps are topological modifications of a parent map. The symmetry of parents is preserved by running these operations. Several operations on maps are known and are currently used to decorate a surface domain. The reader is invited to consult some recent publications in this respect [22]. In the following, only the most important operations will be detailed.

Dual $\mathbf{D u}$ : put a point in the center of each face of the map, next join two such points if their corresponding faces share a common edge [23]. It is the (Poincaré) dual $D u(M)$. The vertices of $D u(M)$ represent faces in $M$ and vice-versa. In the transformed map, the following relations exist: $D u(M) ; v=f_{0} ; e=e_{0} ; f=v_{0}$. Dual of the dual returns the original map: $D u(D u(M))=M$. Tetrahedron is self-dual while the other Platonic polyhedra form pairs: $D u($ Cube $)=$ Octahedron; $D u($ Dodecahedron $)=$ Icosahedron (see Fig. 1 for symbols hereafter used). It is also known the Petrie dual.

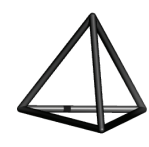

Tetrahedron T $3^{3}$

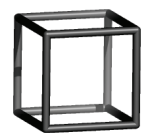

Cube

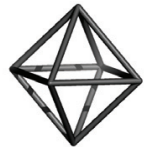

Octahedron

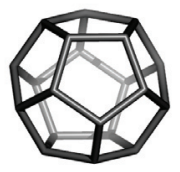

Dodecahedron

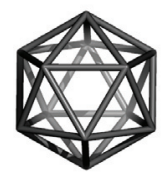

Icosahedron

Do $5^{3}$

Ico $3^{5}$

Fig. 1: The five Platonic polyhedra.

Medial Med: put new vertices in the middle of the original edges and join two vertices if the edges span an angle (and are consecutive). Medial is a 4-valent graph and $\operatorname{Med}(M)=$ $\operatorname{Med}(\operatorname{Du}(M))$, as illustrated in Fig. 2, left. The transformed map parameters are: $\operatorname{Med}(M)$; $v=e_{0} ; e=2 e_{0} ; f=f_{0}+v_{0}$. The medial operation rotates parent $s$-gonal faces by $\pi / s$ [23]. Points in the medial represent original edges, thus this property can be used in topological analysis of edges in the parent polyhedron. Similarly, the points in dual give information on the topology of parent faces. 


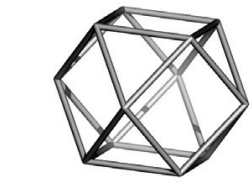

$\operatorname{Med}(C)=$ Cuboctahedron $(3.4)^{2}$

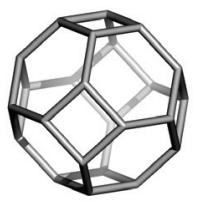

$\operatorname{Tr}(O c t)=$ Truncated Octahedron

$4.6^{2}$

Fig. 2. Medial and Truncation.

Truncation $\boldsymbol{T r}$ : cut off the neighborhood of each vertex by a plane close to the vertex, such that it intersects each edge meeting the vertex. Truncation is similar to the medial, the transformed map parameters being: $\operatorname{Tr}(M) ; \quad v=2 e_{0}=d_{0} v_{0} ; \quad e=3 e_{0} ; f=f_{0}+v_{0}$ [23]. This was the main operation used by Archimedes in building its well-known 13 solids. Fig. 2, right illustrates a transform by this operation.

Polygonal mapping $\boldsymbol{P}_{n}$ : add a new vertex in the center of each face. Put $n$-3 points on the boundary edges. Connect the central point with one vertex on each edge (the end points included). Thus, the parent face is covered by triangles $(n=3)$, quadrilaterals $(n=4)$ and pentagons $(n=5)$. The $P_{3}$ operation is also called stellation or triangulation. The transformed map parameters are: $P_{n}(M) ; v=v_{0}+(n-3) e_{0}+f_{0} ; e=n e_{0} ; f=s_{0} f_{0}$. Fig. 3 gives examples of the $P_{n}$ operations realization [24].

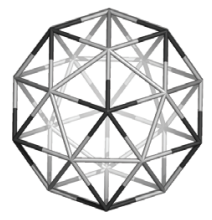

$P_{3}(D o)\left(3^{5}\right)_{3}\left(3^{6}\right)_{5}$

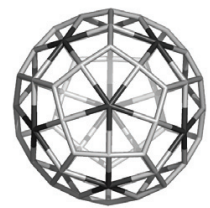

$P_{4}(D o)\left(4^{5}\right)_{3}\left(4^{3}\right)_{5}$

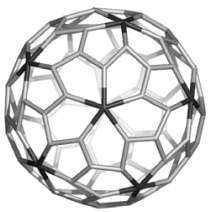

$P_{5}(D o)\left(5^{5}\right)_{3}\left(5^{3}\right)_{5}$

Fig. 3. Polygonal $P_{n}$ operations on the Dodecahedron Do.

Leapfrog Le (tripling) is a composite operation [25-27] that can be written as:

$$
\operatorname{Le}(M)=D u\left(P_{3}(M)\right)=\operatorname{Tr}(D u(M))
$$

A sequence of stellation-dualization rotates the parent $s$-gonal faces by $\pi / s$. Leapfrog operation is illustrated, on a pentagonal face, in Fig. 4. 
A bounding polygon, of size $2 d_{0}$, is formed around each original vertex. In the most frequent cases of 4- and 3-valent maps, the bounding polygon is an octagon and a hexagon, respectively.

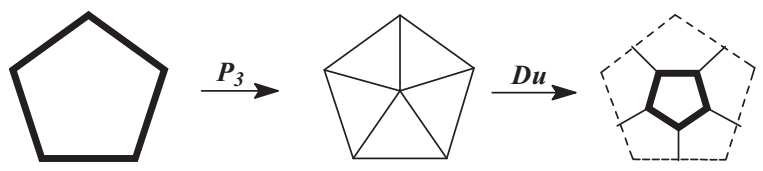

Fig. 4. The Leapfrog Le operation on a pentagonal face

If the map is a $d_{0}$ regular graph, the number of vertices in $L e(\mathrm{M})$ is $\mathrm{d}_{0}$ times larger than in the original map $\mathrm{M}$, irrespective of the tessellation type. Note that in $\operatorname{Le}(M)$ the vertex degree is always 3, as a consequence of the involved triangulation $P_{3}$. In other words, the dual of a triangulation is a cubic net. It is also true that truncation always provides a trivalent lattice. A nice example of using $L e$ operation is: $L e($ Dodecahedron $)=$ Fullerene $\mathrm{C}_{60},\left(5.6^{2}\right)_{60}$.

Quadrupling $\boldsymbol{Q}$ (Chamfering) [25,28] is another composite operation, cf. sequence:

$$
Q(M)=E_{-}\left(\operatorname{Tr}_{P_{3}}\left(P_{3}(M)\right)\right)
$$

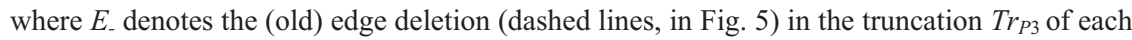
central vertex of the $P_{3}$ operation. The $Q$ operation leaves unchanged the initial orientation of the polygonal faces.

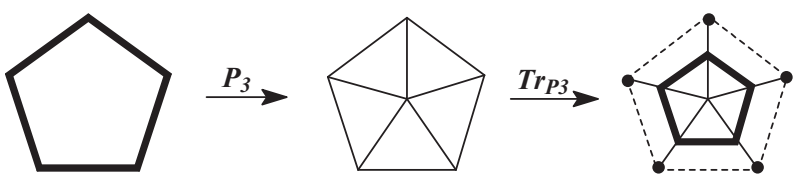

Fig. 5. The Quadrupling $Q$ operation on a pentagonal face.

The vertex multiplication ratio in a $Q$ transformation is $\mathrm{d}_{0}+1$ irrespective of the original map tessellation. $Q$ operation involves two $\pi / s$ rotations, so that the initial orientation of the polygonal faces is preserved. Note that, the quadrupling transform of a 4-valent map is not a regular graph anymore (because of mixing the new trivalent vertices with the parent 4- 
valent ones). Only $Q(M)$ of a 3-valent map is a 3-regular graph. $Q$ insulates the parent faces always by hexagons. An example of this operation is: $Q$ (Dodecahedron) $=$ Fullerene $\mathrm{C}_{80}$, $\left(5.6^{2}\right)_{60}\left(6^{3}\right)_{20}$. It is also called "chamfering" (edge chamfering being equivalent to vertex truncation).

Septupling $S$. Two operations on maps are known: the septupling $\mathrm{S}_{1}$ and $S_{2}$ [24,27,2931].

The $S_{1}$ operation was also called Capra $\mathrm{Ca}$ - the goat, by the Romanian name of the English leapfrog children game [32]. It is a composite operation that can be written as:

$$
S_{1}(M)=\operatorname{Tr}_{P_{5}}\left(P_{5}(M)\right)
$$

with $\operatorname{Tr}_{P 5}$ meaning the truncation of the new, face centered, vertices introduced by $P_{5}$ operation, which involves an $E_{2}$ (i.e., two new points put on each edge) operation.

The nuclearity of the [33] polyhedra is given by:

$$
m=\left(a^{2}+a b+b^{2}\right) ; a \geq b ; a+b>0
$$

which provides the multiplication factor $m=v / v_{0}$. In a 3-valent map, Le $((1,1) ; m=3 ; Q$ $\left((2,0) ; m=4\right.$ and $S\left((2,1) ; m=7\right.$. An example of this operation is: $S_{I}$ (Dodecahedron) $=$ Fullerene $\mathrm{C}_{140},\left(5.6^{2}\right)_{60}\left(6^{3}\right)_{80}$.

$S_{1}$ insulates any face of $M$ by its own hexagons, which are not shared with any old face. It is an intrinsic chiral operation (it rotates the parent edges by $\pi /(3 / 2) s$ and was extensively illustrated in ref. 25. Since $P_{5}$ operation can be done either clockwise or counterclockwise, it results in an enantiomeric pair of objects: $S_{1 S}(M)$ and $S_{1 R}(M)$, with the subscript $S$ and $R$ referring to sinister/rectus stereochemical isomery.

$S_{1}$ can continue with the open operation: $O p_{k}\left(S_{i}(M)\right)$ where $k$ represents the number of points added on the boundary of the parent faces that become the open faces. The resulting open objects have all the polygons of the same $(6+k)$ size. The above operation sequence enables the construction of negatively curved networks. Fig. 6 gives the steps of $S_{1}$ realization on a square face in a trivalent lattice, up to the open structure.

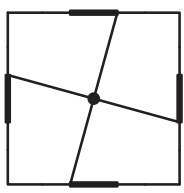

$P_{5}(M)$

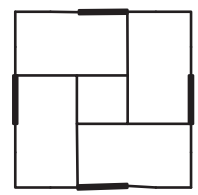

$S_{1}(M)$

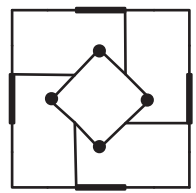

$O p\left(S_{1}(M)\right)$

Fig. 6. Septupling $S_{1}$ operation on a square face, up to the open structure. 
The vertex multiplication ratio in an $\mathrm{S}$ transformation is $2 \mathrm{~d}_{0}+1$ irrespective of the original map tiling.

The $S_{2}$ operation [31] is a simpler one (Fig. 7); it can be achieved by putting four vertices on each edge of the parent map $M\left(E_{4}\right.$ operation) and next join these new vertices in $\operatorname{order}(-1,+3)$ :

$$
S_{2}=J_{(-1,+3)}\left(E_{4}(M)\right)
$$

It insulates the double sized parent faces by pentagons and parent vertices by pentagon $d_{0}$-multiples; the transformed objects are non-chiral ones.

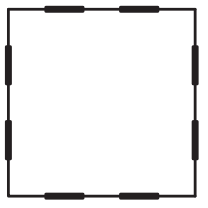

$E_{4}(M)$

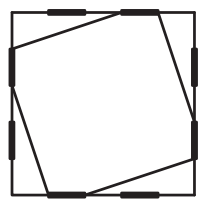

$S_{2}(M)$

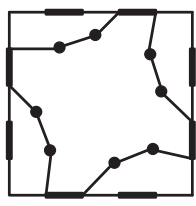

$O p_{2 a}\left(S_{2}(M)\right)$

Fig. 7. Septupling $S_{2}$ operation on a square face, up to the open structure.

Chirality in $S_{2}$ is brought by the $O p$ operation $O p_{2 a}$, achieved by adding two points on alternative edges of the double sized parent face boundary (Fig. 7).

The transformed lattice parameters are identical to those provided by $S_{1}$. Note that both the septupling operations keep the parent vertices. The transformed lattice parameters are: $S_{1}(M) \& S_{2}(M) ; v=v_{0}\left(2 d_{0}+1\right) ; e=7 e_{0} ; f=f_{0}\left(s_{0}+1\right)$. An example of this operation is: $S_{2}$ $($ Tetrahedron $)=$ Fullerene $\mathrm{C}_{28},\left(5^{3}\right)_{4}\left(5^{2} \cdot 6\right)_{24}$.

Peter John [27] has proposed a generalization of operations on maps, inspired from the work of Goldberg [33] and the representation of polyhedra in the $(a, b)$ "inclined coordinates" $\left(60^{\circ}\right.$ between axes).

TOPO GROUP CLUJ has developed four main software programs dedicated to polyhedral tessellation and embedment in surfaces of various genera, either as finite or infinite lattices: TORUS, CageVersatile_CVNET, JSCHEM, OMEGA counter and NanoStudio [34-38]. 


\section{Structure Design}

The hypothetical structures herein discussed were designed by using CVNET and NANO STUDIO. The units used in construction of more complex structures are presented in Fig.8. Five such units, denoted here $\mathrm{U}_{1}$, form a hyper pentagonal ring R5. A dodecahedron having in lieu of its pentagonal faces the hyper-pentagons $\mathrm{R}_{5}$ hyper-faces) is a spongy structure, named in the following $\mathrm{U}_{20}$, to remember the 20 simple cages/units composing it. Next, by identifying the hyper-faces of two units $U_{20}$ one obtains 1-periodic structures. There are experimental data showing that in alloys like AlMn, AlFe, AlCuCo, and AlCoNi, with a diffraction pattern of tenfold rotational symmetry, have also a 1-dimensional translational periodicity along the tenfold rotational axis [39]. This symmetry is also called "axial" symmetry. The rod-like structures herein discussed have been characterized in crystallographic terms by using the TOPOS software [40].

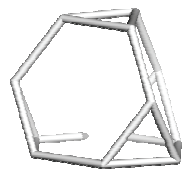

Truncated Tetrahedron TT_12 $\left(3.6^{2}\right)$

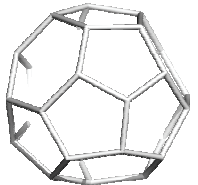

$$
\mathrm{C}_{28} ;\left(5^{3}\right)_{4}\left(5^{2} \cdot 6\right)_{24}
$$

By $\mathrm{S}_{2}(\mathrm{~T})$

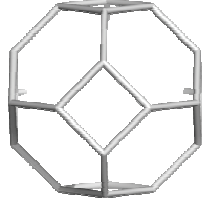

Truncated Octahedron

TO_24 $\left(4.6^{2}\right)$

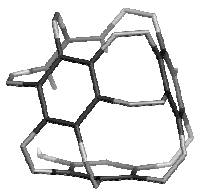

BTA $48\left(6.8^{2}\right)$

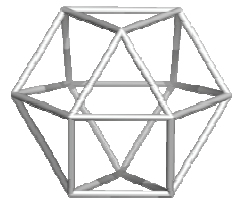

Cuboctahedron

CO_12 (3.4) $)^{2}$

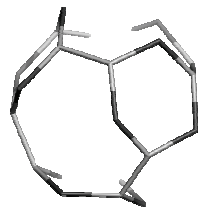

BTZ_24; $\left(6.9^{2}\right)$

By Op(Le(T))

Fig. 8. Units $U_{1}$ used in construction of complex structures

\section{Computational Details}

By the aid of TOPOS (program package for multipurpose crystallochemical analysis [40] we classified the 1-periodic structures assigning them to a space group compatible with the structure and computing the Point Symbol. All the structures are new and never described before in TOPOS databases. 
We used three programs: atom.exe, hinzalign.exe and hin2topos.exe in order to transfer the studied structures to TOPOS.

The program atom.exe enabled to find the direction/orientation of the structure. The three input parameters are: $\mathrm{a}_{0}, \delta, \alpha$, where $\mathrm{a}_{0}$ is an atom of the structure to be the origin of the new coordinates while $\delta$ (metric) and $\alpha$ (angular) are tolerance parameters.

For an atom $\mathrm{a}$, let $\mathrm{E}_{\mathrm{a}}$ be the set of edges incident to a. For two atoms, a, a' we define the "vertex similarity" as the cardinality of $\mathrm{E}_{\mathrm{a}} \cap_{\mathrm{s}} \mathrm{E}_{\mathrm{a}^{\prime}}=\left\{\mathrm{e}_{\mathrm{a}}\left|\mathrm{e}_{\mathrm{a}} \in \mathrm{E}_{\mathrm{a}}, \exists \mathrm{e}_{\mathrm{a}^{\prime}} \in \mathrm{E}_{\mathrm{a}^{\prime}},\right| \mathrm{e}_{\mathrm{a}^{-}} \mathrm{e}_{\mathrm{a}^{\prime}} \mid \leq \delta\right.$; $\left.\angle\left(\mathrm{e}_{\mathrm{a}}, \mathrm{e}_{\mathrm{a}^{\prime}}\right) \leq \alpha\right\}$.

The program collects a set $\mathrm{S}\left(\mathrm{a}_{0}\right)$ of atoms of maximal similarity to $\mathrm{a}_{0}$. The atoms in $\mathrm{S}\left(\mathrm{a}_{0}\right)$ are candidates to be paired with $\mathrm{a}_{0}$ to form the repetitive vector. The vector is chosen according to various criteria, like "shortest", "longest" etc.

Given a vector $v$, the program hinzalign.exe first rotates the coordinate system such that the $\mathrm{z}$ axis becomes parallel to $\mathrm{v}$. The coordinates of each atom are recalculated based on the new (rotated) coordinate system. Next, the coordinate system is translated with the origins in the atom $\mathrm{a}_{0}$ (i.e., in the new (translated) coordinate system, $\mathrm{a}_{0}$ will be $(0,0,0)$ ).

The resulting .hin-file was converted to a TOPOS file using the third program, called hin2topos.exe.

The images obtained with the TOPOS program have to be similar to the original structure in order to be correctly interpreted.

For each structure, the coordination of the nodes (3-c, 4-c and so on), the number of topologically independent nodes ( $n$-nodal), and the Point Symbol (PS) is given. (For the definition of Point Symbol see [41]. The crystallographic data are availble in the supplemenatry as .cif file.

\section{1-Periodic Networks}

In this section, eleven new 1-periodic networks are listed, together with their crystallographic description and the map operation used in their design.

The $\mathrm{U}_{20}$ structures in Fig. 9 and 10 are built up from BTA_48 and BTZ_24 (Fig. 8, bottom row), structures called "polybenzenes" in [42-44]. The unit BTZU ${ }_{20} 480$ was shown to self-arrange in a more complex spherical arrays [45]. 


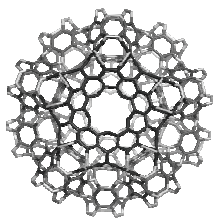

BTAU $_{20 \_} 780$

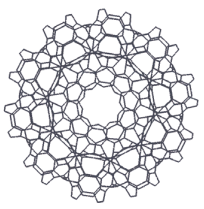

TOPOS view

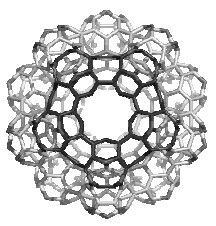

BTZU $20 \_480$

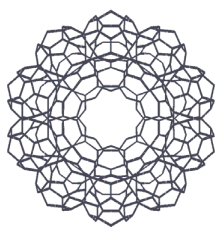

TOPOS view

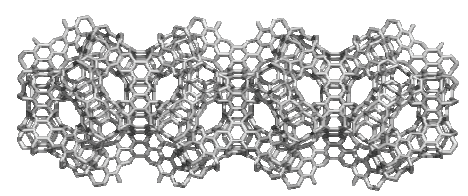

BTA20_4_2490

The unit $\mathrm{U}_{1}$ is BTA_48 (Fig. 8) designed by spanning $L e\left(P_{4}(\mathrm{~T})\right)$

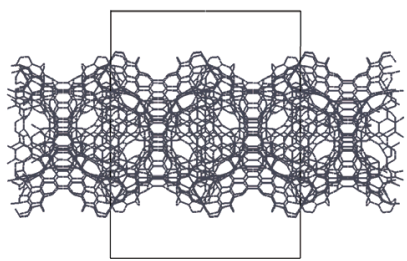

$\mathrm{BTA}_{20} \_$is a 3-c 27-nodal net PS $\left(6.8^{2}\right)_{11}\left(6^{2} .8\right)_{26}\left(6^{3}\right)_{14}$

Fig. 9. $\mathrm{BTA}_{20} \_k$ rod-like structure

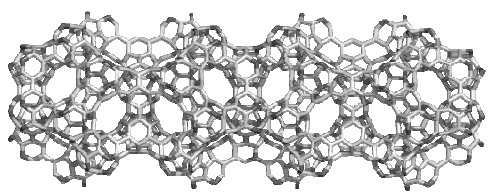

$$
\text { BTZ20_4_1560 }
$$

The unit $\mathrm{U}_{1}$ is BTZ_24 (Fig. 8) designed by spanning $S_{2}(\mathrm{~T})$

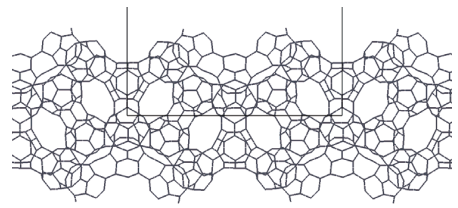

$\mathrm{BTZ}_{20} k$ is 3 -c net 20 -nodal net PS $(5.6 .8)_{2}\left(5.8^{2}\right)_{3}\left(6.8^{2}\right)_{6}$

Fig. 10. BTZ $20 \_k$ rod-like structure 
The unit $\mathrm{TP}_{4} \mathrm{TrsU}_{20} 350$ (Fig. 11) can arrange spherically (as mentioned above for the polybenzene units) or (its tetrapodal units $\mathrm{TP}_{4} \mathrm{Trs}$ 222) translationally, as in the diamond structure [46].

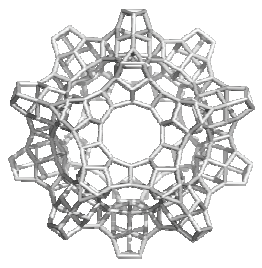

$\mathrm{TP}_{4} \mathrm{TrsU}_{20} \_350$

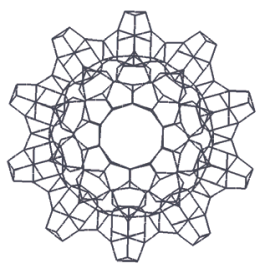

TOPOS view

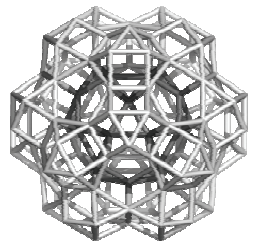

$\mathrm{IDU}_{20} 150$

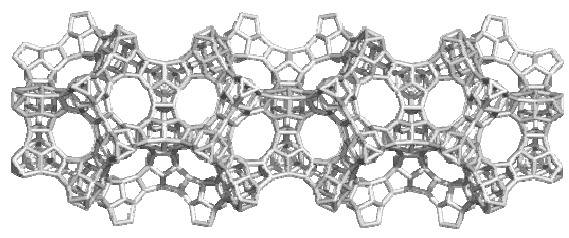

$\mathrm{TP}_{4} \mathrm{Trs}_{20}$ 4_1115

The unit $\mathrm{U}_{1}$ designed by $\operatorname{Trs}\left(P_{4}(\mathrm{~T})\right)$

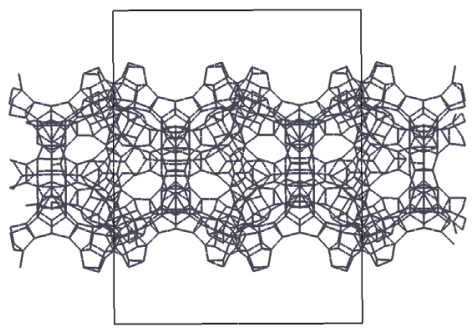

$\mathrm{TP}_{4} \operatorname{Trs}_{20} k$ is 3,4-c 20-nodal net

PS $\left(3.5^{2}\right)_{2}\left(3.5^{4} .8\right)_{5}\left(5^{3}\right)_{4}\left(5^{4} .6 .8\right)_{6}$

Fig. 11. $\mathrm{TP}_{4} \mathrm{Trs}_{20} k$ rod-like structure

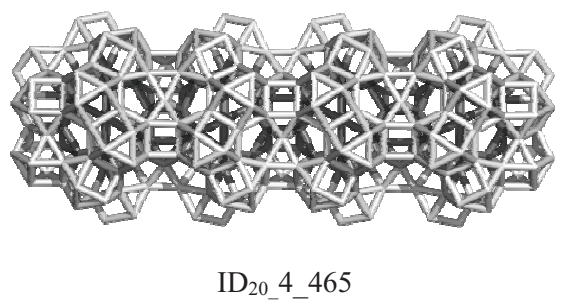

The structure $\mathrm{U}_{20}$ was designed by applying twice the medial Med operation on the centered icosahedron; the core of $\mathrm{U}_{20}$ is the Archimedean Icosidodecahedron (ID) 


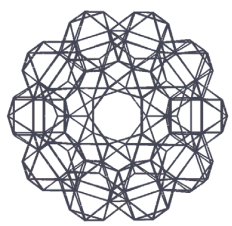

TOPOS view

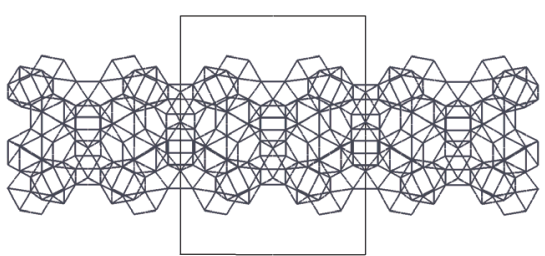

$\operatorname{Med}(\operatorname{Med}(\mathrm{IP}))$

$\mathrm{ID}_{20} k$ is 4,6-c 8-nodal net

$\operatorname{PS}\left(3^{2} \cdot 4^{2} \cdot 5^{2}\right)_{6}\left(3^{3} \cdot 4^{4} \cdot 5^{5} \cdot 6^{3}\right)_{8}\left(3^{3} \cdot 4^{4} \cdot 5^{6} \cdot 6^{2}\right)_{7}$

Fig. 12. ID $_{20} k$ rod-like structure

The structures in Fig. 12 have as the cage unit the Cuboctahedron; the unit IDU $_{20}$ is built up by the sequence $\operatorname{Med}(\operatorname{Med}(\mathrm{IP}))$, the iterative medial operation performed on the centered icosahedron, by operating all the rings. Its core is the Archimedean icosidodecahedron, $\mathrm{ID}=\operatorname{Med}(\mathrm{Ico}) \_30$. Next, $\mathrm{IDU}_{20}$ can form either a rod-like or a sphere-like array or a spherical one [45].

The units $28 \mathrm{U}_{20} 380$ (Fig. 13) and $28 \mathrm{U}_{20} 400$ (Fig. 14) are spongy and filled structures, respectively [45]. They are built up by the small fullerene $\mathrm{C}_{28}$.

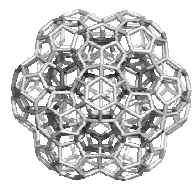

28U $20 \_380$

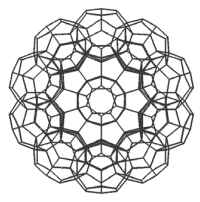

TOPOS view

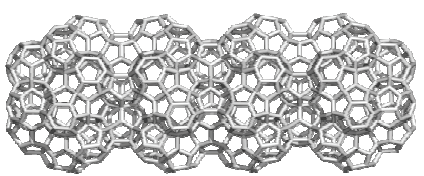

28(380) 20_4_1190

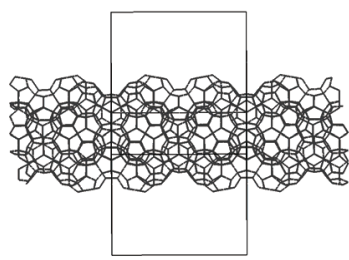

28(380) $)_{20 \_} k$ is 3,4-c 21-nodal net

$$
\operatorname{PS}\left(5^{2} .6\right)_{12}\left(5^{3}\right)_{12}\left(5^{4} \cdot 6.8\right)_{19}\left(5^{5} \cdot 6\right)_{11}
$$

Fig. 13. 28(380) $)_{20} k$ rod-like structure 


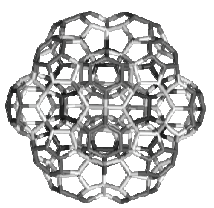

28U $\mathrm{U}_{20} 400$

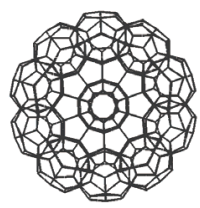

TOPOS view

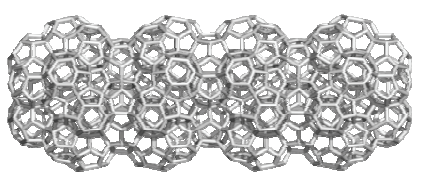

28(400) 20_4_1270

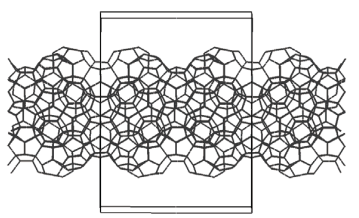

28(400) $)_{20 \_} k$ is 3,4-c 23 nodal net $\operatorname{PS}\left(5^{2} .6\right)_{12}\left(5^{3}\right)_{8}\left(5^{4} \cdot 6.8\right)_{13}\left(5^{5} \cdot 6\right)_{17}\left(5^{6}\right)_{8}$

Fig. 14. 28(400) $)_{20} \_$rod-like structure

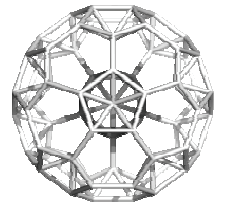

$\mathrm{TT}_{84 \mathrm{U}_{20}}$

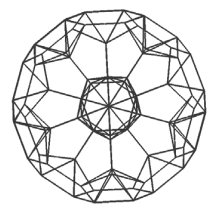

TOPOS view

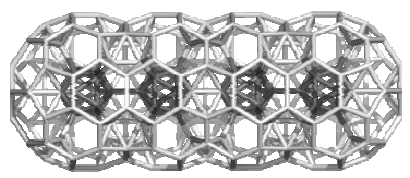

$(\mathrm{TT} 84)_{20}$ 4_240

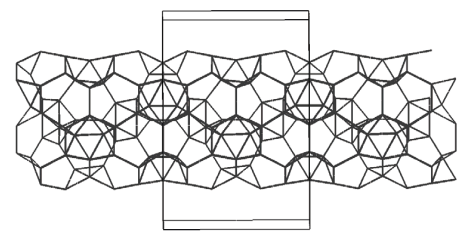

(TT84) $20 \_k$ is 4,5,6-c 5 nodal net $\operatorname{PS}\left(3^{2} \cdot 4 \cdot 6^{3}\right)_{10}\left(3^{3} \cdot 4^{2} \cdot 5 \cdot 6^{4}\right)_{5}\left(3^{5} \cdot 4^{5} \cdot 6^{5}\right)_{11}$

Fig. 15. (TT84) $)_{20 \_} k$ rod-like structure 


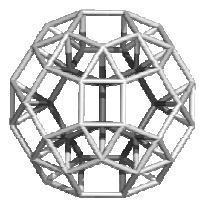

TTDu80U 20

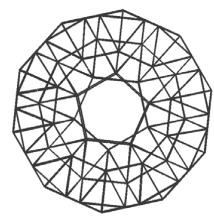

TOPOS view

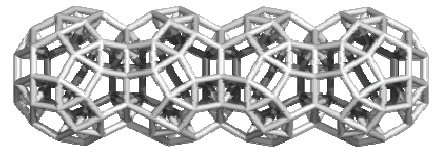

$(\mathrm{TTDu} 80)_{20 \_} 4260$

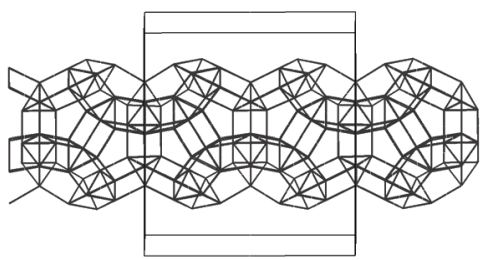

(TTDu80) $20 \_k$ is 5,6-c 5-nodal net $\operatorname{PS}\left(3^{3} \cdot 4^{4} \cdot 5^{3}\right)_{3}\left(3^{3} \cdot 4^{6} \cdot 5^{5} \cdot 6\right)\left(3^{3} \cdot 4^{6} \cdot 5^{6}\right)_{2}$

Fig. 16. $(\text { TTDu } 80)_{20 \_} k$ rod-like structure

The units $\mathrm{TT}_{84} \mathrm{U}_{20}$ (Fig. 15) and $\mathrm{TTDu} 80 \mathrm{U}_{20}$ (Fig. 16) are related to each other and to the icosahedral diamond $[45,47]$. They are based on the truncated tetrahedron TT.

The units 60TOU $20 \_300$ (Fig. 17) and 60TTU $20 \_150$ (Fig. 18) represent aggregations of $\mathrm{C}_{60}[45,48]$

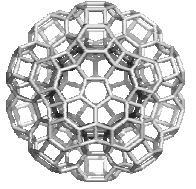

$60 \mathrm{TOU}_{20} 300$

Core $=\mathrm{C}_{60}$

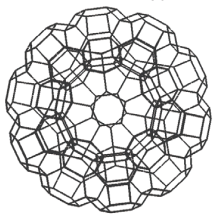

TOPOS view

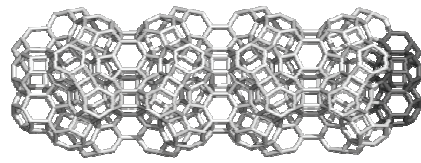

$(60 \mathrm{TO})_{20 \_4} 930$

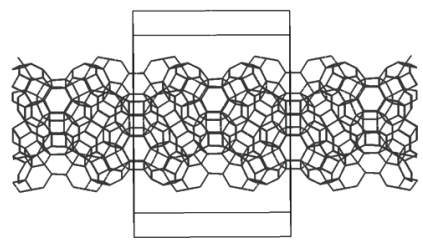

$(60 \mathrm{TO})_{20} k$ is 3,4-c 12-nodal net $\operatorname{PS}\left(4 \cdot 6^{2}\right)_{6}\left(4^{2} \cdot 5 \cdot 6^{3}\right)_{11}\left(4^{2} \cdot 6^{4}\right)_{4}$.

Fig. 17. $(60 \mathrm{TO})_{20} \_$rod-like structure 


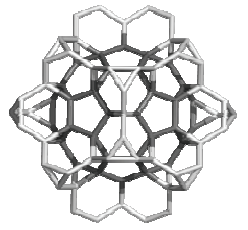

60TTU $20 \_150$

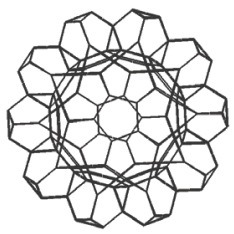

TOPOS view

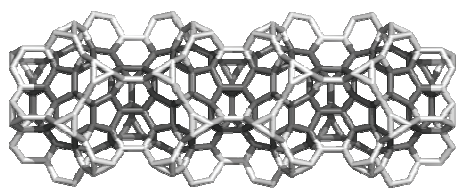

$(60 \mathrm{TT})_{20} 4$ - 465

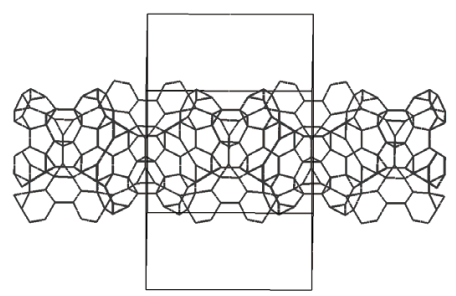

$(60 \mathrm{TT})_{20} \_$is 3,4-c 8-nodal net $\operatorname{PS}\left(3.5 \cdot 6^{4}\right)_{11}\left(3.6^{2}\right)_{6}\left(3 \cdot 6^{4} \cdot 10\right)_{4}$.

Fig. 18. $(60 \mathrm{TT})_{20 \_} k$ rod-like structure

The structure in Figs. 19 and 20 represent substructures and quasicrystals of the diamond $\mathrm{D}_{5}$ $[45,49]$.

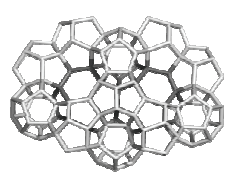

D D $_{\text {Dia }} \sin \_226$

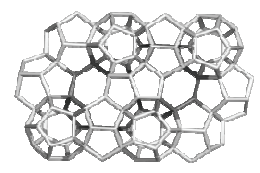

$\mathrm{D}_{5}$ Dia_anti_226

Fig. 19. Substructures of the diamond $\mathrm{D}_{5}$

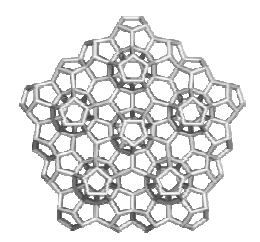

$\mathrm{D}_{5}$ sin_e $524 \_1330$, top

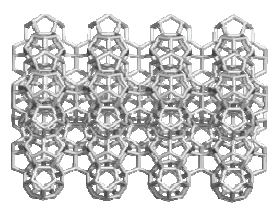

D 5 sin_e524_1330, side

The isomer $\mathrm{D}_{5 \_}$anti is the mtn network, also known as clathrate II $[49,50]$ 

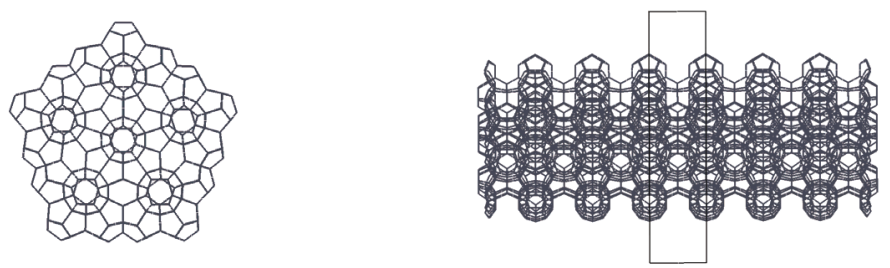

TOPOS view

$$
\begin{gathered}
\mathrm{D}_{5} \_ \text {sin_e } 52 k \text { is } 3,4-\mathrm{c} 27 \text {-nodal net with } \\
\operatorname{PS}\left(5^{3}\right)_{18}\left(5^{5} \cdot 6\right)_{18}\left(5^{5} \cdot 8\right)_{16}\left(5^{6}\right)_{13} .
\end{gathered}
$$

Fig.20. $D_{5} \_$sin_e $52 k$ rod-like structure

\section{Genus Calculation}

In spongy structures, built by tube junctions, the following theorem holds [51]:

Theorem. The genus of a structure, composed from u units, of genus $g_{u}$, is calculated as: $g=u\left(g_{u}-1\right)+1$, irrespective of the unit tessellation.

In fact, the above formula counts the hollows in a rod-like network and was used mainly to check the consistency of a designed structure. It was tested on numerous examples, some of them being presented in Tables 1 to 3 .

In spongy 1-periodic structures bearing closed/finite units, the genus can be calculated from the number of windows (i.e. open faces $f_{o p}$ ): $g=f_{o p}-1$.

In filled 1-periodic structures, the consistency of structures is checked by an extension of Euler-Poincaré formula, due to Schläfli [52]:

$$
f=f_{0}-f_{1}+f_{2}-f_{3}=0
$$

where the sum of figures ( $f_{0}=$ point; $f_{1}=$ edge; $f_{2}=$ face and $f_{3}=$ cage $)$ of a structure equals zero. An example is given in Table 4. 
Table 1. Genus calculation in $\mathbf{2 8}(380)_{20}-k$ (closed) structures (Fig. 13)

\begin{tabular}{cccccccccccc}
\hline $\mathbf{2 8}(380)_{20 \_} k$ & $v$ & $e$ & $f_{5}$ & $f_{6}$ & $f$ & $\chi$ & $g$ & $g_{u}$ & $1)+1$ & & $\mathrm{~g}=f_{o p^{-}}-$ \\
\hline 1 & 380 & 660 & 240 & 20 & 260 & -20 & 11 & - & 11 & 12 & 11 \\
2 & 650 & 1140 & 420 & 30 & 450 & -40 & 21 & 11 & 21 & 22 & 21 \\
3 & 920 & 1620 & 600 & 40 & 640 & -60 & 31 & 11 & 31 & 32 & 31 \\
4 & 1190 & 2100 & 780 & 50 & 830 & -80 & 41 & 11 & 41 & 42 & 41 \\
\hline
\end{tabular}

Table 2. Genus calculation in $(60 \mathrm{TO})_{20} \_k$ rod-like structure (Fig. 17)

\begin{tabular}{ccccccccccc}
\hline$(60 \mathrm{TO})_{20 \_} k$ & $v$ & $e$ & $f_{4}$ & $f_{6}=4 u$ & $f$ & $v-e+f-2$ & $g$ & $u=f_{4} / 6$ & $g_{u}$ & $g=u\left(g_{u}-1\right)+1$ \\
\hline 1 & 300 & 540 & 120 & 80 & 200 & -42 & 21 & 20 & 2 & 21 \\
2 & 510 & 930 & 210 & 140 & 350 & -72 & 36 & 35 & 2 & 36 \\
3 & 720 & 1320 & 300 & 200 & 500 & -102 & 51 & 50 & 2 & 51 \\
4 & 930 & 1710 & 390 & 260 & 650 & -132 & 66 & 65 & 2 & 66 \\
\hline
\end{tabular}

Table 3. Genus calculation in Diamond $\mathrm{D}_{5}$ substructures (Fig. 19)

\begin{tabular}{llllllll}
\hline \multirow{2}{*}{$\begin{array}{l}\text { Structure } \\
v\end{array}$} & $e$ & $g_{u}$ & $u$ & $1)$ & $g=1+u\left(g_{u^{-}} f_{o p}\right.$ \\
\hline 20(12)28(1)ada & 158 & 274 & 1.5 & 4 & 3 & 4 & 3 \\
20(18)28(2)dia_sin 226 & 398 & $2 ; 1.5$ & $3+2$ & 5 & 6 & 5 \\
20(18)28(2)dia_anti226 & 398 & 1.5 & 8 & 5 & 6 & 5 \\
\hline
\end{tabular}

Table 4. Space filling figures calculation in rod-like (TT84) $20 \_k$ structures cf. $f=f_{0}-f_{1}+f_{2}-f_{3}=0$ (Fig. 15)

\begin{tabular}{lllllllll}
\hline$k$ & Atoms & $v$ & $e$ & $r_{3}$ & $r_{6}$ & 2 & \multicolumn{2}{c}{$u=v-e+r f$} \\
\hline 1 & 84 & 84 & 192 & 80 & 50 & 20 & 22 & 0 \\
2 & 136 & 136 & 323 & 140 & 85 & 36 & 38 & 0 \\
3 & 188 & 188 & 454 & 200 & 120 & 52 & 54 & 0 \\
4 & 240 & 240 & 585 & 260 & 155 & 68 & 70 & 0 \\
\hline
\end{tabular}




\section{Conclusions}

Structures showing 1-periodicity can be associated to quasicrystal networks having axial symmetry. We focused here on the construction of such hypothetical structures by the aid of map operations (as implemented in CVNET software) in designing the repeating units and a builder (Nano Studio) in assembling them in large 1-periodic networks. Topology of these structures was presented in crystallographic terms. Also the genus calculation vas rationalized function of the net parameters.

Acknowledgments. Virginia R. Bucila acknowledges that this work was possible with the financial support of the Sectorial Operational Programme for Human Resources Development 2007-2013, co-financed by the European Social Fund, under the project number POSDRU/107/1.5/S/76841 with the title „Modern Doctoral Studies: Internationalization and Interdisciplinarity".

Supporting Materials. The structural data in .cif format for all the 11 1-periodic structures here described.

\section{References}

[1] F. C. Frank, J. S. Kasper, Complex alloy structures regarded as sphere packings. Definitions and basic principles, Acta Cryst. 11 (1958) 184-190.

[2] G. Benedek, L. Colombo, Hollow diamonds from fullerenes, Mater. Sci. Forum 232 (1996) 247-274.

[3] M. Deza, M. I. Shtogrin, Octahedrites, Symmetry Culture Sci. 11 (2003) 27-64.

[4] X. Blasé, G. Benedek, M. Bernasconi, Structural, mechanical, and superconducting properties of clathrates, in: L. Colombo, A. Fasolino (Eds.), Computer-Based Modeling of Novel Carbon Systems and Their Properties - Beyond Nanotubes, Springer, Dordrecht, 2010, pp. 171-206.

[5] A. Haji-Akbari, M. Engel, A. S. Keys, X. Zheng, R. G. Petschek, P. Palffy-Muhoray, S. C. Glotzer, Disordered, quasicrystalline and crystalline phases of densely packed tetrahedra, Nature 462 (2009) 773-777.

[6] A. L. Mackay, A dense non-crystallographic packing of equal spheres, Acta Cryst. 15 (1962) 916-918.

[7] H. S. M. Coxeter, Close-packing and so forth, Illinois J. Math. 2 (1958) 746-758.

[8] H. S. M. Coxeter, Close packing of equal spheres, in: H. S. M. Coxeter (Ed.), Introduction to Geometry, Wiley, New York, 1961, pp. 405-411.

[9] M. Goldberg, On the densest packing of equal spheres in a cube, Math. Mag. 44 (1971) 199-208.

[10] C. Zong, J. Talbot, Sphere Packings, Springer, New York, 1999.

[11] T. C. Hales, The sphere packing problem, J. Comput. Appl. Math. 44 (1992) 41-76. 
[12] T. C. Hales, Historical overview of the Kepler conjecture, Discr. Comput. Geom. 36 (2006) 5-20.

[13] M. Gardner, The Colossal Book of Mathematics: Classic Puzzles, Paradoxes, and Problems, Norton, New York, 2001, pp. 128-136.

[14] T. C. Hales, A proof of the Kepler conjecture, Ann. Math. 162 (2005) 1065-1185.

[15] S. T. Hyde, S. Ramsden, Chemical frameworks and hyperbolic tilings, in: P. Hansen, P. Fowler, M. Zheng (Eds.), Discrete Mathematical Chemistry, Am. Math. Soc., Providence, 2000, pp. 203-224.

[16] A. H. Schoen, Infinite periodic minimal surfaces without self-intersections, NASA Technical Note D-5541, 1970.

[17] M. V. Diudea, M. Petitjean, Symmetry in multi tori, Symmetry Culture Sci. 19 (2008) 285-305.

[18] O. Bonnet, Note sur la therorie generale des surfaces, C. R. Acad. Sci. Paris 37 (1853) 529-532.

[19] L. Euler, Elementa doctrinae solidorum, Novi Comm. Acad. Scient. Imp. Petrop. 4 (1758) 109-160.

[20] F. Harary, Graph Theory, Addison-Wesley, Reading, 1969.

[21] C. Baerlocher, W. H. Meier, D. H. Olson, Atlas of Zeolite Framework Types, Elsevier, Amsterdam, 2007.

[22] M. V. Diudea, Nanomolecules and Nanostructures - Polynomials and Indices, Univ. Kragujevac, Kragujevac, 2010.

[23] T. Pisanski, M. Randić, Bridges between geometry and graph theory, in: C. A. Gorini (Ed.), Geometry at Work, Math. Assoc. Am., Washington, 2000, pp. 174-194.

[24] M. V. Diudea, C. L. Nagy, Periodic Nanostructures, Springer, Dordrecht, 2007.

[25] V. Eberhard, Zur Morphologie der Polyeder, Teubner, Leipzig, 1891.

[26] P. W. Fowler, How unusual is $\mathrm{C}_{60}$ ? Magic numbers for carbon clusters, Chem. Phys. Lett. 131 (1986) 444-450.

[27] M. V. Diudea, M. Ştefu, P. E. John, A. Graovac, Generalized operations on maps, Croat. Chem. Acta 79 (2006) 355-362.

[28] M. V. Diudea, P. E. John, Covering polyhedral tori, MATCH Commun. Math. Comput. Chem. 44 (2001) 103-116.

[29] M. V. Diudea, Covering forms in nanostructures, Forma 19 (2004) 131-163.

[30] M. V. Diudea, Covering nanostructures, in: M. V. Diudea (Ed.), Nanostructures-Novel Architecture, Nova, New York, 2005, pp. 203-242.

[31] M. V. Diudea, Nanoporous carbon allotropes by septupling map operations, J. Chem. Inf. Model. 45 (2005) 1002-1009.

[32] M. V. Diudea, Capra - a leapfrog related operation on maps, Studia Univ. "BabesBolyai" 48 (2003) 3-16.

[33] M. Goldberg, A class of multi-symmetric polyhedral, Tôhoku Math. J. 43 (1937) 104108.

[34] M. V. Diudea, B. Parv, O. Ursu, Torus, Babes-Bolyai Univ., Cluj, 2003.

[35] M. Stefu, M. V. Diudea, CageVersatile_CVNET, Babes-Bolyai Univ., Cluj, 2005.

[36] C. L. Nagy, M. V. Diudea, JSChem, Babes-Bolyai Univ., Cluj, 2005.

[37] S. Cigher, M. V. Diudea, Omega Counter, Babes-Bolyai Univ., Cluj, 2006. 
[38] C. L. Nagy, M. V. Diudea, NANO-Studio, Babes-Bolyai Univ., Cluj, 2009.

[39] L. Bendersky, Quasicrystal with one-dimensional translational symmetry and a tenfold rotation axis, Phys. Rev. Lett. 55 (1985) 1461-1463.

[40] V. A. Blatov, Multipurpose crystallochemical analysis with the program package TOPOS, IUCr Comp. Comm. Newslett. 7 (2006) 4-38. http://www.topos.samsu.ru .

[41] V. A. Blatov, M. O'Keeffe, D. M. Proserpio, Vertex-, face-, point-, Schläfli-, and Delaney-symbols in nets, polyhedra and tilings: recommended terminology, Cryst. Eng. Comm. 12 (2009) 44-48.

[42] B. Szefler, M. V. Diudea, Polybenzene revisited, Acta Chim. Slov. 59 (2012) 795-802.

[43] M. O'Keeffe, G. B. Adams, O. F. Sankey, Predicted new low energy forms of carbon, Phys. Rev. Lett. 68 (1992) 2325-2328.

[44] B. Szefler, O. Ponta, M. V. Diudea, Energetics of polybenzene multi tori, J. Mol. Struct. (Theochem) 1022 (2012) 89-93.

[45] M. V. Diudea, Quasicrystals: between spongy and full space filling, in: M. V. Diudea, C. L. Nagy (Eds.), Diamond and Related Nanostructures, Springer, Dodrecht, 2013.

[46] M. V. Diudea, A. Ilić, All-pentagonal face multi tori, J. Comput. Theor. Nanosci. 8 (2011) 736-739.

[47] J. Breza, M. Kadlečikova, M. Vojs, M. Michalka, M. Vesely, T. Danis, Diamond icosahedron on a TiN-coated steel substrate, Microelectron. J. 35 (2004) 709-712.

[48] A. Bende, M. V. Diudea, Energetics of multi-shell cages, in: M. V. Diudea, C. L. Nagy (Eds.), Diamond and Related Nanostructures, Springer, Dodrecht, 2013.

[49] M. V. Diudea, C. L. Nagy, All pentagonal ring structures related to the C20 fullerene: Diamond D5, Diam. Relat. Mater. 23 (2012) 105-108.

[50] M. O'Keeffe, M. A. Peskov, S. J. Ramsden, O. M. Yaghi, The reticular chemistry structure resource (RCSR) database of, and symbols for crystal nets, Acc. Chem. Res. 41 (2008) 1782-1789.

[51] M. V. Diudea, B. Schefler, Nanotube junctions and the genus of multi-tori, Phys. Chem. Chem. Phys. 14 (2012) 8111-8115.

[52] L. Schläfli, Theorie der vielfachen Kontinuität Zürcher und Furrer, Zürich, 1901 (Reprinted in: Ludwig Schläfli, 1814-1895, Gesammelte Mathematische Abhandlungen, Band 1, 167-387, Verlag Birkhäuser, Basel, 1950) 